\title{
How do porosity-inducing techniques affect antibiotic elution from bone cement? An in vitro comparison between hydrogen peroxide and a mechanical mixer
}

\author{
K. Shiramizu • V. Lovric $\cdot$ A. Leung $\cdot$ W.R. Walsh
}

Received: 7 September 2007 / Accepted: 8 January 2008 / Published online: 17 March 2008

\begin{abstract}
Background Increasing the porosity of an antibioticloaded cement spacer increases the antibiotic elution, but the correlation between porosity and antibiotic elution is not well documented. The purposes of this study was to attempt new porosity-increasing methods and to investigate the correlation between antibiotic elution and both total and surface porosity.

Materials and methods Five types of antibiotic-loaded bone cement (ALBC) using $2 \mathrm{~g}$ cefazolin and $40 \mathrm{~g}$ cement were prepared. Other than manual mixing, hydrogen peroxide was used as a foaming agent and a mixing drill piece was used as a mechanical device to try to induce porosity when mixing the cement. Elution of antibiotic into phosphate-buffered saline was measured from $1 \mathrm{~h}$ to 1 week. Surface porosity was calculated from density values which were measured with a density kit and an electronic balance, while total porosity was quantified using microcomputed tomography.

Results When a mixing drill piece was used to induce porosity, we observed a significant increasin antibiotic elution compared to a manually mixed ALBC. On the
\end{abstract}

K. Shiramizu (凶) $\cdot$ V. Lovric $\cdot$ A. Leung $\cdot$ W.R. Walsh

Surgical \& Orthopaedic Research Laboratories

Prince of Wales Hospital, University of New South Wales

Level 1, Clinical Sciences Building, Avoca Street, Randwick,

NSW Australia 2031

e-mail: keishiramizu@gmail.com other hand, hydrogen peroxide reduced the elution significantly. Mild correlation between the total amount of cluted in 1 week antibiotic elution and total porosity was observed.

Conclusions In terms of improving elution, the mixing drill piece seemed to be efficient. A relationship between surface porosity and elution efficacy was not observed.

Keywords Antibiotic-loaded bone cement - Antibiotic elution · Arthroplasty $\cdot$ Deep infection

\section{Introduction}

Deep infection following total joint arthroplasty is considered to be one of the most severe complications [1-5]. Antibiotic-loaded bone cement (ALBC) is an accepted and efficient method of local antibiotics delivery and is widely used for prophylaxis and treatment of deep infection after arthroplasty and other orthopedic surgeries [6-8]. The elution characteristics of ALBC and antibiotics have been investigated in many previous papers [9-15]. When ALBC is used for fixing the prosthesis in the primary arthroplasty, the most important point is to obtain rigid fixation as it is well recognized that bone cement is plagued with a number of drawbacks that contribute to the aseptic loosening of cemented arthroplasties. To achieve the initial rigid and long endurable fixation with reducing the porosity of the cement, various cementing techniques such as antibiotic mixing technique, cement gun, negative pressure mixing (vacuum mixing) [16, 17], and centrifugation [17-19] are currently performed. Moreover, in order to maintain the cement's mechanical strength, a low-dose ALBC product ( $1 \mathrm{~g}$ antibiotic per $40 \mathrm{~g}$ bone cement) is available for the initial fixation of the prosthesis as prophylaxis; the 
mechanical property of this low-dose ALBC is stronger than the hand-mixed one and as strong as nonantibiotic control cement $[6,20]$. Meanwhile, in treating infected arthroplasties, elution efficacy of the antibiotics is most important when ALBC is used for fabricating the joint spacer. In this circumstance, the mechanical strength of ALBC is not so important. It had also been reported that increasing the ALBC porosity increases the antibiotic elution, so dextran and glycine were used as space fillers to obtain a more porous ALBC $[12,21]$. In those studies, antibiotic elution properties were examined in detail, and a significantly higher elution efficacy was found when ABLC contained space filler. However, detail of cement porosity and other methods of obtaining higher porosity of ALBC were not described in these previous studies. When considering the cement pores as pathways that enable the antibiotics to spread outside the cement spacer, surface porosity of the cement spacer will be the most appropriate indicator for the elution efficacy because the pores which are located deep inside the spacer without any junction to the outside are considered to have no relation with antibiotic elution. The question remains: how deep must the pores be within the cement spacer in order not to release relevant amounts of antibiotic? This remains hard to quantify.

Recently McLaren et al. [22] reported that evaluated the permeability or the ease by which fluids pass through a structure is directly dependant on the porosity of that structure. They used a visualization method with phenolphthalein to evaluate the permeability but also mentioned that its quantitative evaluation was difficult. Furthermore, the antibiotic elution related to surface and total porosity was not well documented.

In this study, mechanical and chemical mixing approaches were attempted in order to obtain higher porosity ALBC. Furthermore, the effect of surface and total porosity of ALBC on the elution characteristic curve over a short time period was investigated.

\section{Materials and methods}

\section{ALBC sample preparation}

To study the effect of surface porosity on antibiotic elution efficacy from bone cement, an in vitro elution study was designed using five different preparations of ALBC.

Powdered cefazolin sodium was obtained from Mayne Pharma (Australia) and used in all samples. Two types of bone cements were used: normal viscosity cement (Simplex P; Howmedica, Limerick, Ireland) and low viscosity cement (CMW 3; DePuy, Blackpool, UK). One package of Simplex $P$ contains $40.0 \mathrm{~g}$ finely divided powder and $20 \mathrm{ml}$ of a colorless liquid. The composition of the powder, by weight, is
$75 \%$ methyl methacrylate (styrene copolymer), $15 \%$ polymethyl methacrylate (PMMA), and 10\% barium sulfate; the liquid monomer is $97.5 \%$ methyl methacrylate (monomer), $2.5 \% \quad N, N$-dimethyl- $p$-toluidine and $0.0075 \%$ hydroquinone. One package of CMW 3 contains $40 \mathrm{~g}$ finely divided powder and $18.37 \mathrm{ml}$ of a colorless liquid. The composition of the powder, by weight, is $88.85 \%$ PMMA, $2.05 \%$ benzoyl peroxide, and $9.1 \%$ barium sulfate; the liquid monomer is $98.215 \%$ methyl methacrylate, $0.816 \%$ $N, N$-dimethyl-p-toluidine, $0.945 \%$ ethyl alcohol, $0.022 \%$ ascorbic acid, and $0.002 \%$ hydroquinone.

Each type of ALBC was preparated using $2 \mathrm{~g}$ cefazolin and $40 \mathrm{~g}$ cement powder ( $n=5$ per type). One ALBC type was made with manual mixing but to increase the porosity of the specimens, a cement mixing drill piece was devised (Fig. 1) and was used as a mechanical foaming method. Moreover, $6 \%$ hydrogen peroxide $\left(\mathrm{H}_{2} \mathrm{O}_{2}\right)$ was used as a chemical foaming agent. Thus, the 5 ALBC preparations investigated were:

- Type A. Simplex P cefazolin (open bowl hand mixing)

- Type B. Simplex $\mathrm{P}$ cefazolin $+5 \mathrm{ml} \mathrm{6 \%} \mathrm{H}_{2} \mathrm{O}_{2}$ (open bowl hand mixing)

- Type C. CMW 3 cefazolin $+5 \mathrm{ml} 6 \% \mathrm{H}_{2} \mathrm{O}_{2}$ (open bowl hand mixing)

- Type D. Simplex P cefazolin (mixed with cement mixing drill piece for $3.5 \mathrm{~min}$ in open bowl)

- Type E. Simplex P cefazolin $+5 \mathrm{ml} 6 \% \mathrm{H}_{2} \mathrm{O}_{2}$ (mixed with cement mixing drill piece for $3.5 \mathrm{~min}$ in open bowl) To prepare all types of ALBC, the antibiotic and cement powder were mixed thoroughly while dry. The liquid monomer was then added with thorough mixing in accordance with the manufacturer's instructions. An exothermic polymerization reaction followed, resulting in liquid cement. When used, $\mathrm{H}_{2} \mathrm{O}_{2}$ was added at this point. Mixing was continued until a dough-like mass was formed that did not stick to the surgical gloves. After mixing, each ALBC preparation was packed into a steel mould that contained 25 individual 6-mm diameter, 12$\mathrm{mm}$ height holes. From the 25 samples, 10 most uniform specimens were picked for each group. Five were used

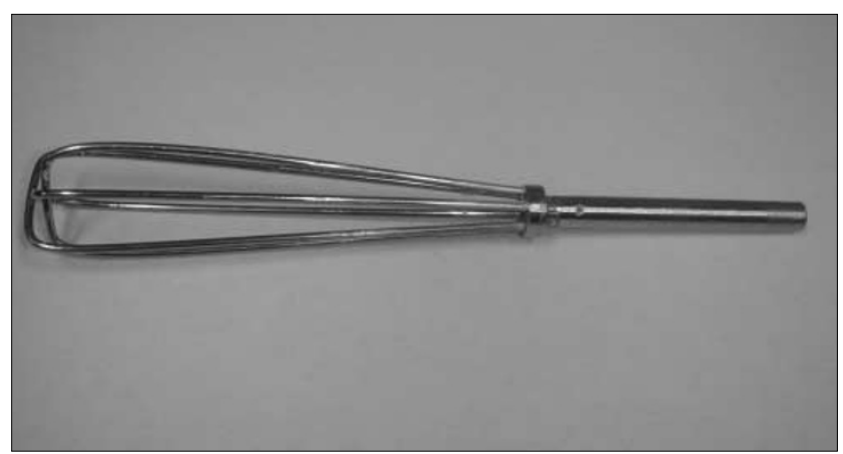

Fig. 1 Cement mixing drill piece. The length of the whisk is $90 \mathrm{~mm}$ and maximum diameter is $21 \mathrm{~mm}$ 
for surface and total porosity measurement and five for elution testing. It was packed into the mould from one side to reduce the number of air pockets and to keep the consistency of the samples as even as possible. Once the mould was filled, a flat steel plate was placed on either side and the assembly was compressed with a clamp. Excess cement was removed from the sides. The mixing and manipulation process was $4 \mathrm{~min}$ in duration, according to the manufacturers' specifications. The samples were left to cure for $30 \mathrm{~min}$. After curing, the samples were removed from the mould and both ends were polished with 600-grid sand paper.

In the hand mixing method (types A, B, C), the mixture was randomly stirred with an inert spatula at about 2 strokes/s, while types D and E were mixed using the cement mixing drill piece which was mounted to a cordless drill. Speed was 1200 rotations per minute (RPM).

\section{Elution testing}

Each cement specimen was fully immersed in $5 \mathrm{ml}$ phosphate buffered saline (PBS) at room temperature in a covered container. At the designated sampling intervals, each specimen was removed from the container, rinsed with $10 \mathrm{ml}$ normal saline and placed in a fresh $5-\mathrm{ml}$ bath in a fresh container. A container from each of the cement specimen at each of the time intervals 1, 24, 48, 96 and $168 \mathrm{~h}$ was stored at $-20^{\circ} \mathrm{C}$ until they were analyzed. Cefazolin concentrations were determined by high-performance liquid chromatography [23], with the following modification to the original protocol. For sample preparation, Sep Pack Alumina B cartridges (Waters, Milford, MA, USA) were used. The liquid chromatograph was equipped with a $50 \mu \mathrm{l}$ sample loop and a Spherisorb-ods1 column (Deeside Ind. Est., Queensferry, UK). The mobile phase used was $0.277 \% \mathrm{Na}_{2} \mathrm{PO}_{4}+0.186 \%$ citric acid:acetonitryl (90:10). SMAD Data System for Apple Macintosh Computers (SMADChrom v2.2, Morgan Kennedy Research, TX, USA) was used to record the data. The concentration of cefazolin in each PBS sample was determined from a calibration curve. The total amount of antibiotic eluted from each specimen was calculated by summing up data from all time points.

\section{Surface porosity measurement}

Surface porosity trends were qualitatively determined by comparing the measured bulk density (dry mass of natural, undisturbed material per unit volume) of the cylindrical sample with the theoretical particle density (mass per unit volume of the solid particles, without the voids) of standard PMMA bone cement $\left(1.21 \mathrm{~g} / \mathrm{cm}^{3}\right)$ [24].
Bulk density was determined using a density measurement kit and an electronic balance. The mass of each ALBC cylinder was measured in air and in water. The theoretical volume of the particle was determined by subtracting the sample mass in water from the sample mass in air. Bulk density was calculated to be the sample mass in air divided by the theoretical volume of the particle.

\section{Total porosity measurement}

Total porosity was measured using a micro-computed tomography (micro-CT) system (Skyscan 1072; Skyscan, Aartselaar, Belgium). In brief, the high-resolution microCT uses the cone-beam reconstruction method to account for the conical geometry of the X-ray source. It enables 3dimensional reconstruction and analysis of an object to be generated from 2-dimensional X-ray shadow images of the object from different angular views. The projections are captured as the object rotates on a high-precision stage. The desktop Skyscan Micro-CT system uses an Xray $C C D$ camera with a resolution of 1024 x 1024 pixels.

Each ALBC sample was placed on the rotational stage and was translated along the continuously variable magnification stage to achieve the desired resolution $(7.8 \mu \mathrm{m})$. The sample was then rotated through $180^{\circ}$ and images were captured at $80 \mathrm{kV}, 100 \mu \mathrm{A}$ every $0.9^{\circ}$. Repeated scans were performed in the beginning of the experiments to verify the reproducibility of the method. The noise of the resulting grey-scale images was removed with a lowpass aluminium filter. Once all images were captured, 300 slices were reconstructed using the standard Skyscan software package (NRECON, Skyscan).

Porosity was quantified by the use of another Skyscan software package (CTAn v.1.5.0, Skyscan). From one of the reconstructed slices, a region of interest was established and interpolated to all the remaining 299 slices. Within the program, the threshold of the image was adjusted, consistently throughout all the samples analyzed, so that the pores were dark while the rest of the image was white and represented solid objects for the subsequent analysis. Thus, the program differentiated the pores in the image, performed a void counting, and determined the pore area, the total area, and the ultimately the percent porosity of the selected section of the sample.

The Tukey-Kramer post-hoc test was used to determine the significance of differences in total porosity and surface porosity measurements and total antibiotic elution amount, achieved with ALBC prepared with different mixing methods and cements. Significance was accepted at $p<0.05$. Furthermore in order to investigate the correlation between different porosity measurements and the total amount of antibiotic eluted by the samples, Pearson's coefficient was calculated. 


\section{Results}

The time course of cefazolin elution from 5 types of ALBC is illustrated in Fig. 2. ALBC type D had the highest elution rate from the first hour to day 2 , while type A exhibited the highest elution rate from the day 4 to week 1 .

In total porosity measurement, there was no significant difference among the ALBC preparations (Fig. 3a). However, there were significant differences between types A and D, A and E, B and E, C and E, and C and D in surface porosity measurements (Fig. 3b). Regarding total antibiotic elution (Fig. 3c), types A and D were best

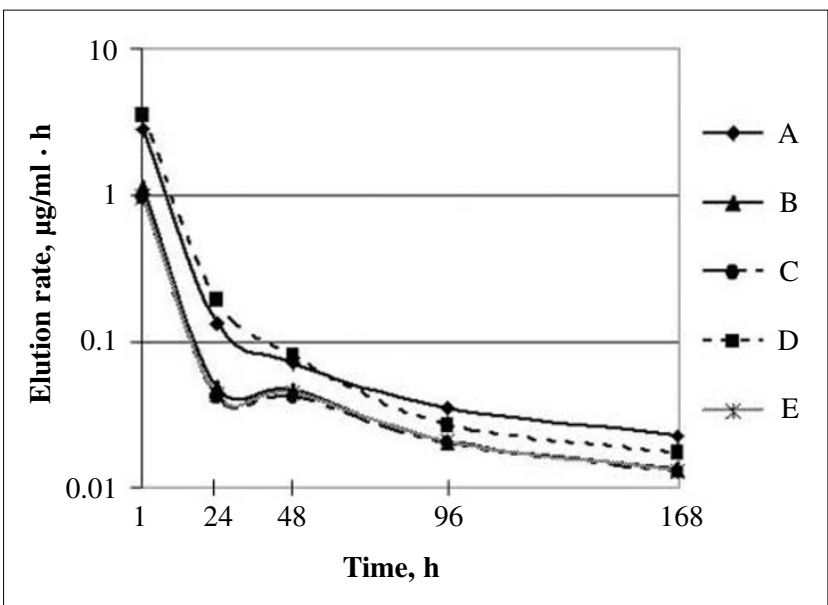

Fig. 2 Time course of cefazolin release for five antibiotic-loaded bone cement (ALBC) preparations. Values are means of determinations each and are plotted on a logaritmic scale. ALBC types A and D, prepared without $\mathrm{H}_{2} \mathrm{O}_{2}$, had a higher elution rates throughout the 1-week testing period even though types D eluted significantly more antibiotic than type $\mathrm{A}(p=0.006)$.

Moderate significant correlation between total porosity and total antibiotic eluted was observed $(p=0.0237, \mathrm{r}=0.448)$ (Fig. 4a). In contrast, the expected correlation between the surface porosity and total antibiotic elution amount was not observed ( $p=0.7813, \mathrm{r}=-0.059)$ (Fig. 4B).

\section{Discussion}

In this study, $6 \%$ hydrogen peroxide was used as a chemical foaming agent. In previous reports, glycine and dextran were documented as space fillers which facilitated antibiotic elution $[12,21]$. Our purpose of adding hydrogen peroxide to bone cement was to create plenty of foam while cement mixing took place. This foam, generating gas, was expected to enlarge the pores in the cement as well as create new pores, ultimately resulting in increased porosity within the specimen. The increased number of pores was expected to act as pathways for antibiotic to leach out from the inside and in turn increase the antibiotic elution rate. In this study, ALBC preparations B, C and E, made with hydrogen peroxide, showed much lower antibiotic elution than those without it. As we mixed the hydrogen peroxide with the bone cement during its liquid phase, the foam may have been reduced by the weight of the liquid bone cement. Apart from not increasing porosity as expected, hydrogen peroxide seemed to inhibit the elution of the antibiotic from the cement spacers. Although ALBC types D and E had similar total porosi-
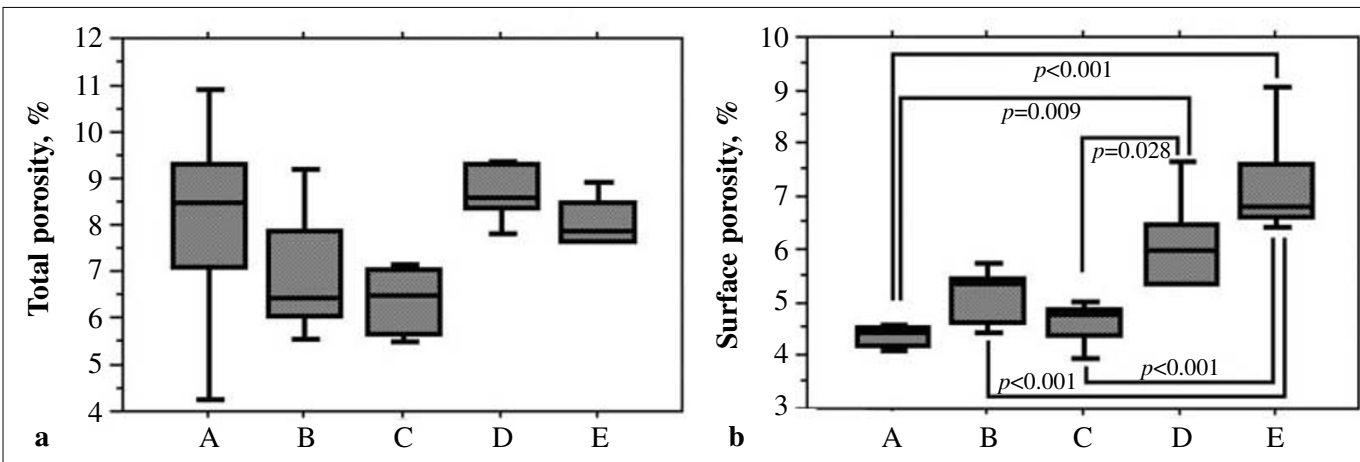

Fig. 3a-c Porosity and elution properties of five ALBC preparations. All data are displayed in box and whisker plots. a Total porosity: no differences between groups are significant. b Surface porosity: Type A (control) has the lowest surface porosity, which is significantly less than that of types D and E. c Total amount of antibiotic eluted in 1 week. Type D eluted the most antibiotic, significantly more than type A which had the second highest elution amount 


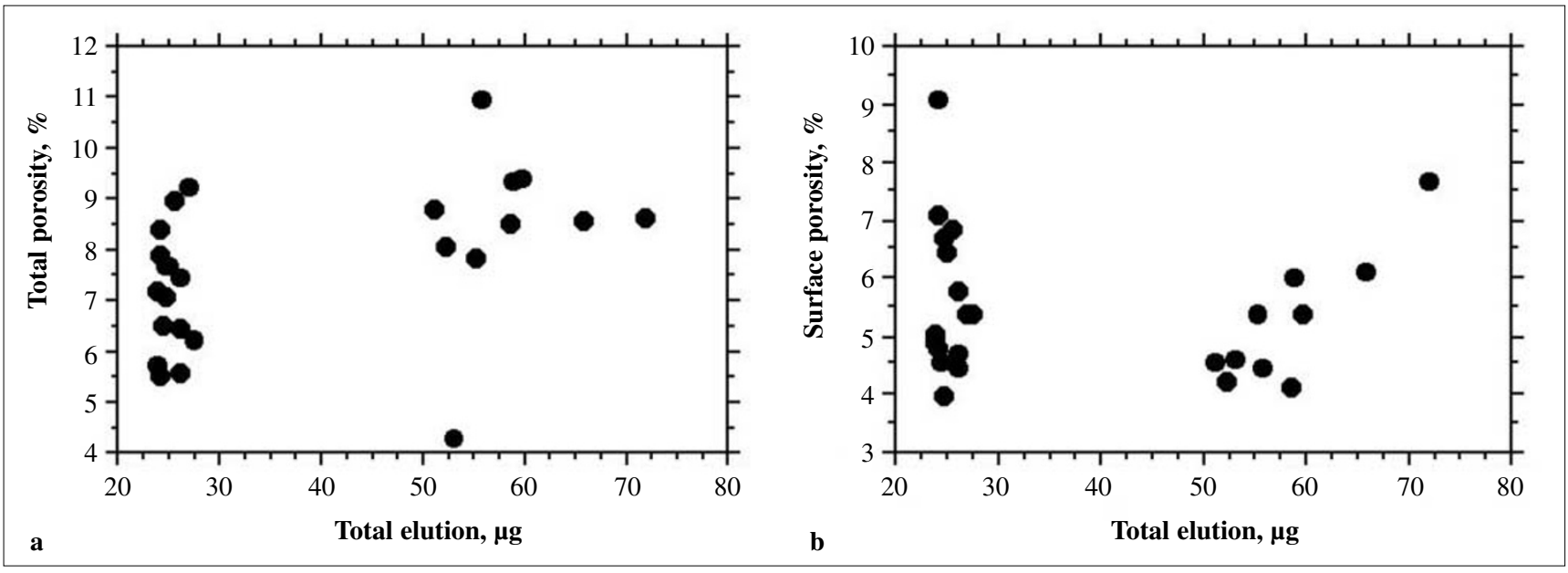

Fig. 4a, b Correlation between porosity and total elution amount. Total elution amount was calculated by adding all the elution data obtained in one week per sample. Shown are individual values for all 5 samples in each ALBC preparation. a Moderate correlation was observed between the total porosity and total elution $(p=0.0237, \mathrm{r}=0.448)$. b No correlation was observed between surface porosity and total elution amount $(p=0.7813, \mathrm{r}=-0.059)$

ties (mean, $8.627 \%$, and $7.889 \%$ respectively), type E had a much lower elution rate than type D. Also, the total amount of cefazolin released over 1 week was much lower for type E $(24.822 \mu \mathrm{g})$ than for type D $(59.683 \mu \mathrm{g})$. The only difference in preparation of the two ALBC types was the inclusion of $5 \mathrm{ml} 6 \% \mathrm{H}_{2} \mathrm{O}_{2}$ in $\mathrm{E}$.

The cement mixing drill piece was used to prepare both ALBC types D and $\mathrm{E}$ in hope of producing foam and increasing porosity in the same manner as an egg beater would. The rotational speed was $1200 \mathrm{rpm}$, almost the same as that used in the operating theater. The surface porosity measurements were directly calculated from the obtained bulk density value. This value was considered to be directly affected by the permeability of ALBC or the ease by which fluids pass through the ALBC samples, which is highly dependant on the surface porosity. The less dense the material, the higher the porosity of the material will be, enabling more fluids to get into the surface pores and cracks and hence achieve higher elution rates. In the comparison of ALBC types A and D, the latter showed a higher total antibiotic elution. In regard to the porosity, the surface porosity of type D was higher than that of type A, while there was no significant difference in the total porosity. As mentioned earlier, it is very hard to quantify how deep the pores must be within the cement spacer in order not to release any antibiotic. Consequently, had the samples been left in water for longer than a minute during density measurements it is possible that they would have soaked up more water and a difference between the surface porosity of the two groups would have not been significant as the micro-CT data indicated within total porosity measurements. This claim is supported by McLaren et al. who measured the permeability of ALBC [22]. In their report, higher perme- ability was shown in 30-day samples than in 2-day ones. Therefore, our immersing period might have been too short to evaluate the surface porosity. In terms of elution measurements, we also measured elution after 2 and 4 weeks but the quantities were so slight that they were excluded from this study. As also documented in other papers, the ALBC spacer does not elute a substantial amount for 30 days; therefore it may be difficult to establish the immersing period to measure the surface porosity related the elution efficacy. Nonetheless, in terms of antibiotic elution over a short period of time (1 week), the mixing drill piece is useful.

It is generally accepted that medium Paracos- $\mathrm{R}$ (medium viscosity cement, Smith \& Nephew, Memphis, USA) has more efficient elution characteristics than other cement types [7, 8]. The elution ability of Paracos-R was reported to be twice that of Simplex P and CMW 3, while the elution characteristics of Simplex P and CMW 3 are reported to be similar [7]. In order to eliminate the factor of the cement quality itself and any effect that it might have on the study, we used the latter two products to compare the effects that different viscosity cements would have on antibiotic elution efficacy. ALBC types B and C were used for this comparison. Although type B (normal viscosity cement Simplex P) had a slightly higher antibiotic elution amount than type $\mathrm{C}$ (low viscosity cement, CMW 3), this difference was not significant.

In our measurement of total porosity through microCT, we found a moderate correlation with total antibiotic elution. This finding supports previous reports which claimed that increasing porosity increases antibiotic elution $[12,21]$. Surface porosity would be a direct indicator of the elution efficacy from ALBC theoretically, but its measurement related to elution efficacy is still controver- 
sial. Therefore, from this study, we consider that the total porosity may still be a reliable guide to elution efficacy.

The main purpose of this study was to improve antibiotic elution by increasing the porosity and not to just increase the porosity. In terms of obtaining a better elution, the mixing drill piece seemed to be an efficient method. In this study, hydrogen peroxide inhibitored foam creation. If dextran or glycine type space fillers, which have previously been proven to increase elution rates $[12,21]$. are mixed with bone cement with our cement mixing device, it may be possible to obtain a more favorable elution efficacy.

Conflict of interest statement No benefits in any form have been received or will be received from any commercial party related directly to the subject of this article.

\section{References}

1. Bourne RB (2004) Prophylactic use of antibiotic bone cement: an emerging standard - in the affirmative. J Arthroplasty 19:69-72

2. Diefenbeck M, Mückley T, Hofmann GO (2006) Prophylaxis and treatment of implant-related infections by local application of antibiotics. Injury 37:S95-S104

3. Garvin KL, Hanssen AD (1995) Infection after total hip arthroplasty: past, present, and future. J Bone Joint Surg Am 77:1576-1588

4. Mont MA, Waldman BJ, Hungerford DS (2000) Evaluation of preoperative cultures before second-stage reimplantation of a total knee prosthesis complicated by infection: a comparisongroup study. J Bone Joint Surg Am 82:1552-1557

5. Tetsworth K (2003) Infection after total knee arthroplasty: evaluation and treatment. Curr Opin Orthop 14:45-51

6. Hanssen AD, Spangehl MJ (2004) Practical applications of antibiotic-loaded bone cement for treatment of infected joint replacements. Clin Orthop Relat Res 427:79-85

7. Penner MJ, Duncan CP, Masri BA (1999) The in vitro elution characteristics of antibiotic-loaded CMW and Palacos-R bone cement. J Arthroplasty 14:209-214

8. Trippel SB (1986) Current concepts review: antibiotic-impregnated cement in total joint arthroplasty. J Bone Joint Surg Am 68:1297-1302
9. Adams K, Couch L, Cierny G et al (1992) In vitro and in vivo evaluation of antibiotic diffusion from antibiotic-impregnated polymethylmethacrylate beads. Clin Orthop Relat Res 278:244-252

10. Anguita-Alonso P, Rouse MS, Piper KE et al (2006) Comparative study of antimicrobial release kinetics from polymethylmethacrylate. Clin Orthop Relat Res 445:239-244

11. Jiranek W (2005) Antibiotic-loaded cement in total hip replacement: current indications, efficacy, and complications. Orthopedics 28:s873-s877

12. Kuechile DK, Landon GC, Musher DM, Noble PC (1991) Elution of vancomycin, daptomycin, and amykacin from acrylic bone cement. Clin Orthop Relat Res 264:302-308

13. Penner MJ, Masri BA, Duncan CP (1996) Elution characteristics of vancomycin and tobramycin combined in acrylic bone-cement. J Arthroplasty 11:939-944

14. Seldes RM, Winiarsky R, Jordan LC et al (2005) Liquid gentamicin in bone cement: a laboratory study of a potentially more costeffective cement spacer. J Bone Joint Surg Am 87:268-272

15. Stevens CM, Tetsworth KD, Calhoun JH, Mader JT (2005) An articulated antibiotic spacer used for infected total knee arthroplasty: a comparative in vitro elution study of Simplex and Palacos bone cements. J Orthop Res 23:27-33

16. Lewis G, Austin GE (1994) Mechanical properties of vacuummixed acrylic bone cement. J Appl Biomater 5:307-314

17. Macaulay W, DiGiovanni CW, Restrepo A et al (2002) Differences in bone-cement porosity by vacuum mixing, centrifugation, and hand mixing. J Arthroplasty 17:569-575

18. Hansen D, Jensen JS (1992) Mixing does not improve mechanical properties of all bone cements. Acta Orthop Scand 63:13-18

19. Jasty M, Davies JP, O'Connor DO et al (1990) Porosity of various preparations of acrylic bone cements. Clin Orthop Rel Res 259:122-129

20. Hanssen AD (2004) Prophylactic use of antibiotic bone cement: an emerging standard - in opposition. J Arthroplasty 19:73-77

21. McLaren AC, Nelson CL, McLaren SG, DeClerk GR (2004) The effect of glycine filler on the elution rate of gentamicin from acrylic bone cement. Clin Orthop Relat Res 427:25-27

22. McLaren AC, Nelson CL, McLaren SG, Wassell DL (2005) Phenolphthalein used to assess permeability of antibiotic-laden polymethylmethacrylate. Clin Orthop Relat Res 439:48-51

23. Liang D, Chow D, White C (1994) High-performance liquid chromatic assay of cefazolin in rat tissues. J Chromatogr B Biomed Appl 656:460-465

24. Vallo CI, Montemartini PE, Fanovich MA (1999) Polymethylmethacrylate-based bone cement modified with hydroxyapatite. J Biomed Mater Res 48:150-158 